MATEC Web of Conferences 13,01001 (2014)

DOI: $10.1051 /$ matecconf/ 20141301001

(C) Owned by the authors, published by EDP Sciences, 2014

\title{
AutoGuide System for Conventional Vehicle on Highway
}

\author{
Rosmawati Mat Zain
}

Department of Mechanical Engineering, Universiti Teknologi PETRONAS, Bandar Seri Iskandar, 31750 Tronoh, Perak, Malaysia

\begin{abstract}
This paper highlights possible development of driver assistance control called AutoGuide system for conventional vehicle. The basic aim is to simulate the AutoGuide system which helps the conventional vehicle to be maneuvered on highway. The system is simulated using MATLAB SIMULINK software which accommodate the control of the vehicle steering and vehicle speed. The results are presented in five case studies: 1) Straight road condition, 2) Curve road condition, 3) Ramp road condition, 4) Sharp corner road condition and 5) Road with small to large size foreign object. It is shown that the system give expected responses.
\end{abstract}

\section{Introduction}

Driving Assistance is a technology that has been used for many years by most developed vehicle producer. Among the oldest driving assistance that is widely used today is power steering. Power Steering reduces the efforts needed to turn the steering wheel and makes maneuvering at low speed [1]. In most recent development, the power steering system is further improved to Electric Power Assisted Steering (EPAS). The previous system is using hydraulic motor power which powered by the vehicle engine thus reduce the efficiency of the vehicle. While in EPAS the steering completely uses electric motor to assist the steering and power source from the generated electric by the engine itself.

Leader of vehicle manufacturer, Volkswagen had developed the driving assistance system called Automated Distance Regulator (ADR). The system works by mounting three-beam distance sensor to monitor the area in-front of the vehicle that will display information for the drivers to interpret and take action based on his judgment [2]. This system helps the driver to know the speed of the vehicle in front of him/her, and regulate the vehicle velocity by controlling the throttle, transmission, and brake.

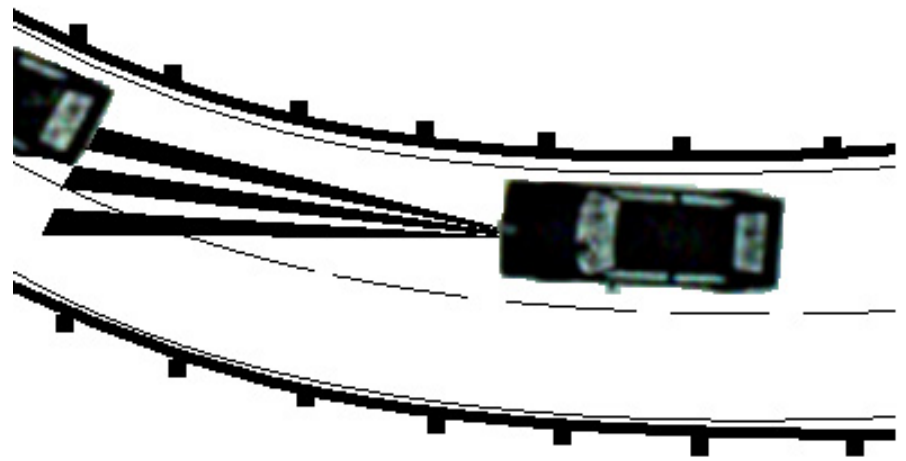

Figure 1. Automated Distance Regulation (ADR).

This is an Open Access article distributed under the terms of the Creative Commons Attribution License 2.0, which permits unrestricted use, distribution, and reproduction in any medium, provided the original work is properly cited. 
After this early development by Volkswagen there is an urge to create new driving assistance which is still under development and gone for countless time of trial and experiment such as Autonomous Intelligent Cruise Control (AICC). The main purpose of AICC is to examine the flow of the traffic and then compare its performances with the human driver model [3]. In the later future AICC is hope to become a full functioning system that might replace the driver, making it an artificial driver. BMW has a fraction key of this technology so called Cruise Control. The cruise control is capable of driving a vehicle without need of human driver by controlling the throttle, transmission and brake. The system will automatically regulate everything to the preset value input by the driver, while the driver only needs to control the steering to maneuver the vehicle.

Due to the flood of this new driving assistance technology, there is a suggestion of how this system can be tested in real application with the most controlled environment. This study is then explained in the paper of Automated Highway System (AHS). The paper objective is concern with the possibilities of combination of certain system that available today to take over the human essentials in driving [4]. The paper also stresses on the lacking of communication between the current development with the driver and another system.

Among the importance concern that must be overcome by the driver assistant system is to avoid the incoming obstacle along the road profile. This is further discussed in the chapter of Unmanned Vehicle Control [5]. The variables that are needed to be control for obstacle avoidance are the lateral control, longitudinal control and steering control. Hence this stresses the importance of sensors technology.

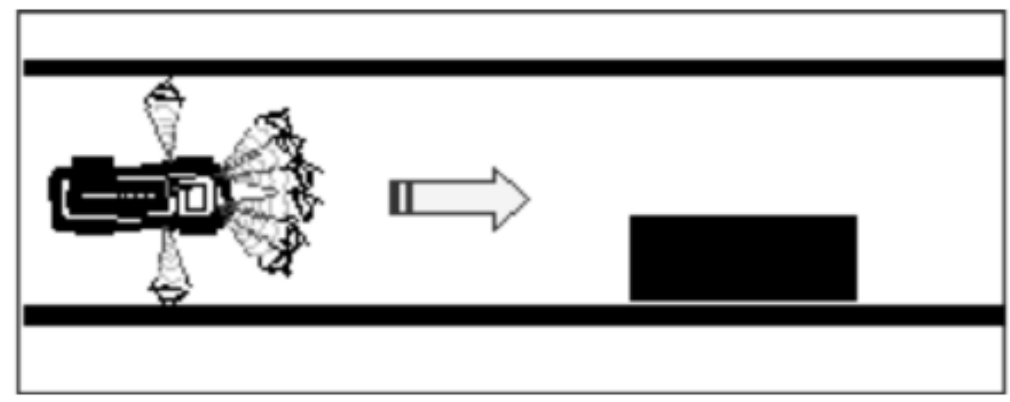

Figure 2. Unmanned vehicle on road with obstacle.

Sachin Modi [6] discusses the type of sensors for obstacle avoidance and three installation methods for different kind of sensor and its reaction towards obstacle. The objective is to see how these three sensors guide the model through several trial of obstacle on road. Each sensor is analyzed in terms of their performances, reliability, advantages and disadvantages.

\section{AutoGuide System}

AutoGuide is a system that assists the driver to control the vehicle steering and also to regulate the vehicle velocity. This is done by monitoring the distance between the vehicle and the highway divider. Highway environment is chosen because the distance monitoring system cannot work in normal road. The highway divider is a set of reference point to indicate the road profile. From the logic sequence of the system, it will then give instruction based on the distance monitoring for the vehicle steering control and vehicle speed regulation. Conventional vehicle is highlighted because of the importance of this system to enhance the safety features of the conventional vehicle. The issues regarding conventional vehicle is the deficiency cause by the human limitation due to long journey travelled.

The flow chart show how the distance monitoring system became the main function to determine the next sequence for the system to control the vehicle steering and regulate the vehicle velocity. 

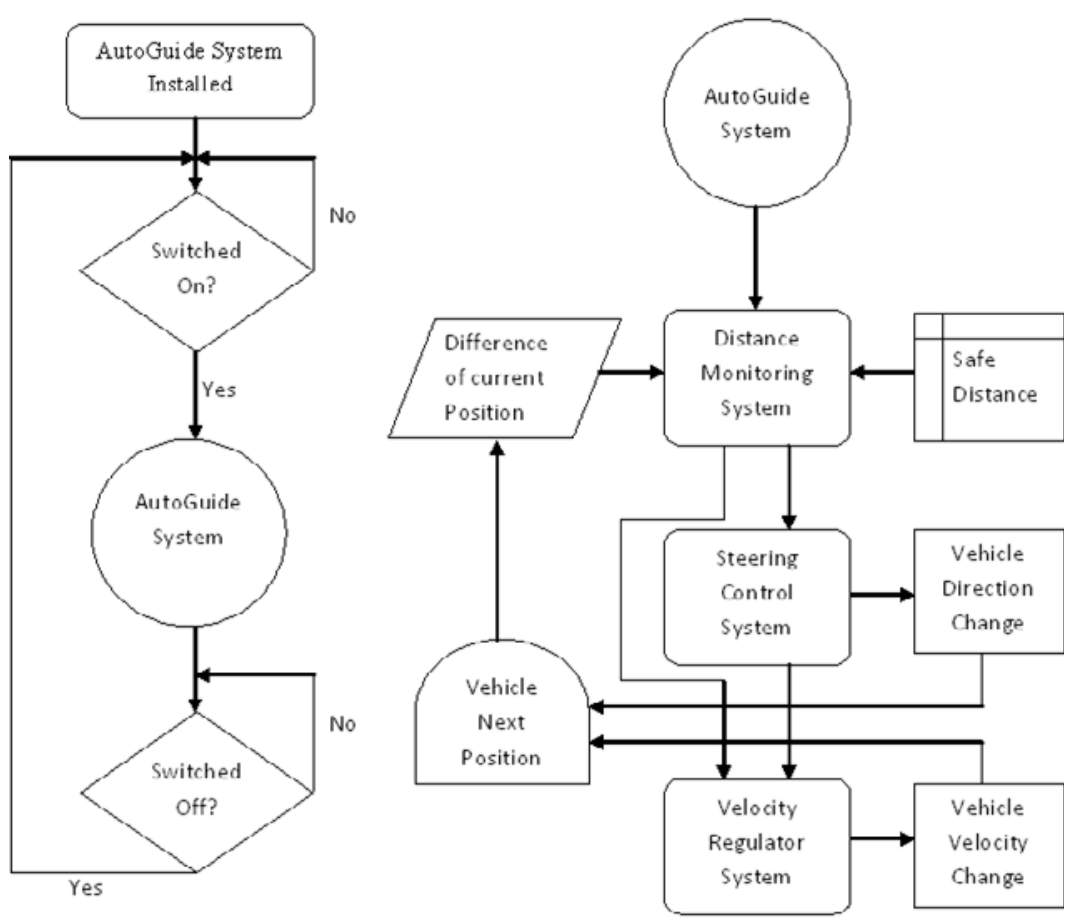

Figure 3. AutoGuide system flow chart.

The sensor will emit and receive signal to give the position of the vehicle from the highway divider. This data will then be differentiated from the safety factor for the system to operate. It will then determine which direction the steering should be rotated, either approaching or away from the divider. This data is also compared to the data before to determine whether is it appropriate to increase or reduce the vehicle speed.

\section{System Design}

AutoGuide system can be divided into two major systems as this system is controlling two variables; 1) steering control and 2) vehicle speed.

The first input is from the steering control loop. The input will be read as negative if the vehicle is approaching the highway divider and positive if it is away from the highway divider. The system will constantly give new input with a frequency of 10 data per second. This data is then compared to the safe distance from the divider which is set to 1.5 meters. If the comparison element gives positive value indicating the vehicle is far away from the highway divider, the stepper motor will rotate the steering to turn into direction of approaching the highway divider and inversely vice versa.

The second input is from the distance monitoring system where positive value means the vehicle is in safe position and negative means the vehicle is no longer in safe position. Based on these two arguments the velocity regulator will increase or reduce the vehicle velocity. Change of direction from the distance monitoring and the steering control can be manipulated to indicate if the system is approaching the highway divider too fast or vice versa. Therefore this data can be used to be manipulated for the vehicle velocity control.

A few simplifications and assumptions are made in the system design which are;

1. Road profile is a controlled environment with highway divider always exists along the way.

2. The vehicle is simplified to single track vehicle and the dynamic of the actual vehicle is negligible.

3. The steering of vehicle, the vehicle throttle, transmission, and brake system for vehicle velocity control are controllable by the system. 


\section{Simulation and Results}

The main input is the Road Profile which is preset as Straight Road, Curve Road, Ramp Road, Sample Road and Test Road. Sample Road is the sample of road profile taken from the actual fraction of road from Ipoh to Kuala Lumpur. The Test Road is a condition of road with foreign object in between the vehicle and the highway divider, divided into three type of foreign object; small size object, medium size object and large size object. The road profiles will be read by the sensor and then compare with the position of the vehicle at that current time. The difference of this two reference point will be then compared with the safe distance to determine which direction the steering should be steered. The action of the system to turn the steering is then kept in the memory of the vehicle velocity control loop for reference on the next loop.

Velocity regulation system will determine the vehicle velocity by increasing the speed to the maximum allowable speed or reduced until the vehicle stop. The speed of the vehicle is regulated for an increase or decrease in a range of 0.1 meters positive and negative area. The vehicle will come to a sudden stop when the difference between the vehicle and the highway divider is less than 0.1 meter.

Five different environment cases have been simulated in MATLAB/SIMULINK as below:

Case 1: Straight Road Simulation

Case 2: Curve Road Simulation

Case 3: Ramp Road Simulation

Case 4: Sharp Corner Road Simulation

Case 5: Road with Small to Large Size Foreign Object

Case 1, 2, 3 and 4 are presented in Figure 8, which is also representing a fraction of highway road that exist between Ipoh and Kuala Lumpur. Simulation shows that the system is capable to follow the road profile according to the case 1,2 , and 3 . When the road profile changes drastically as in case 4 , the system is no longer be able follow the road profile but instead it will take safety measure to avoid the highway divider by making a sudden stop.

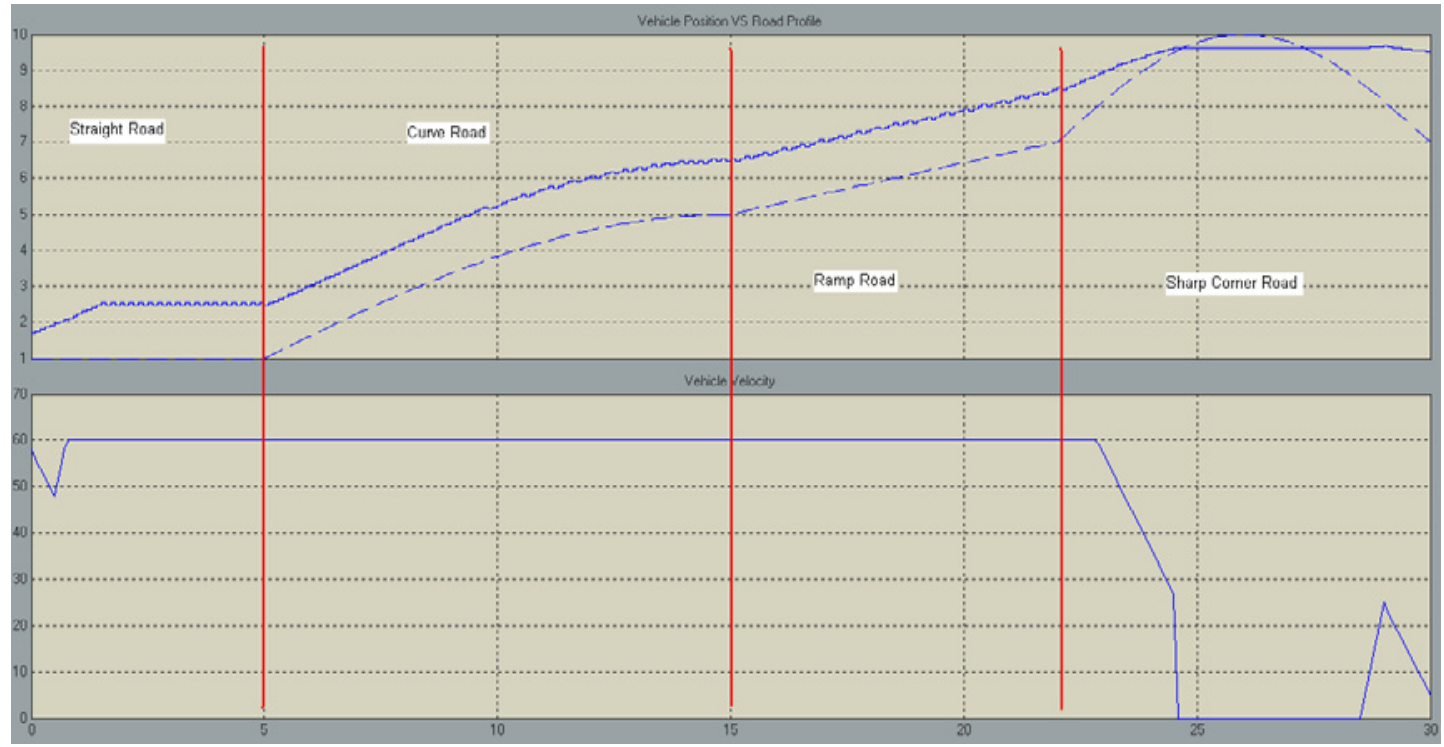

Figure 4. Simulation results based on studied road.

Case 5 in Figure 9 show the simulation of the system to react to the foreign object sized from small to large. This is comparable to the real object on highway road such like cones, motorcyclist and construction with temporary divider. 


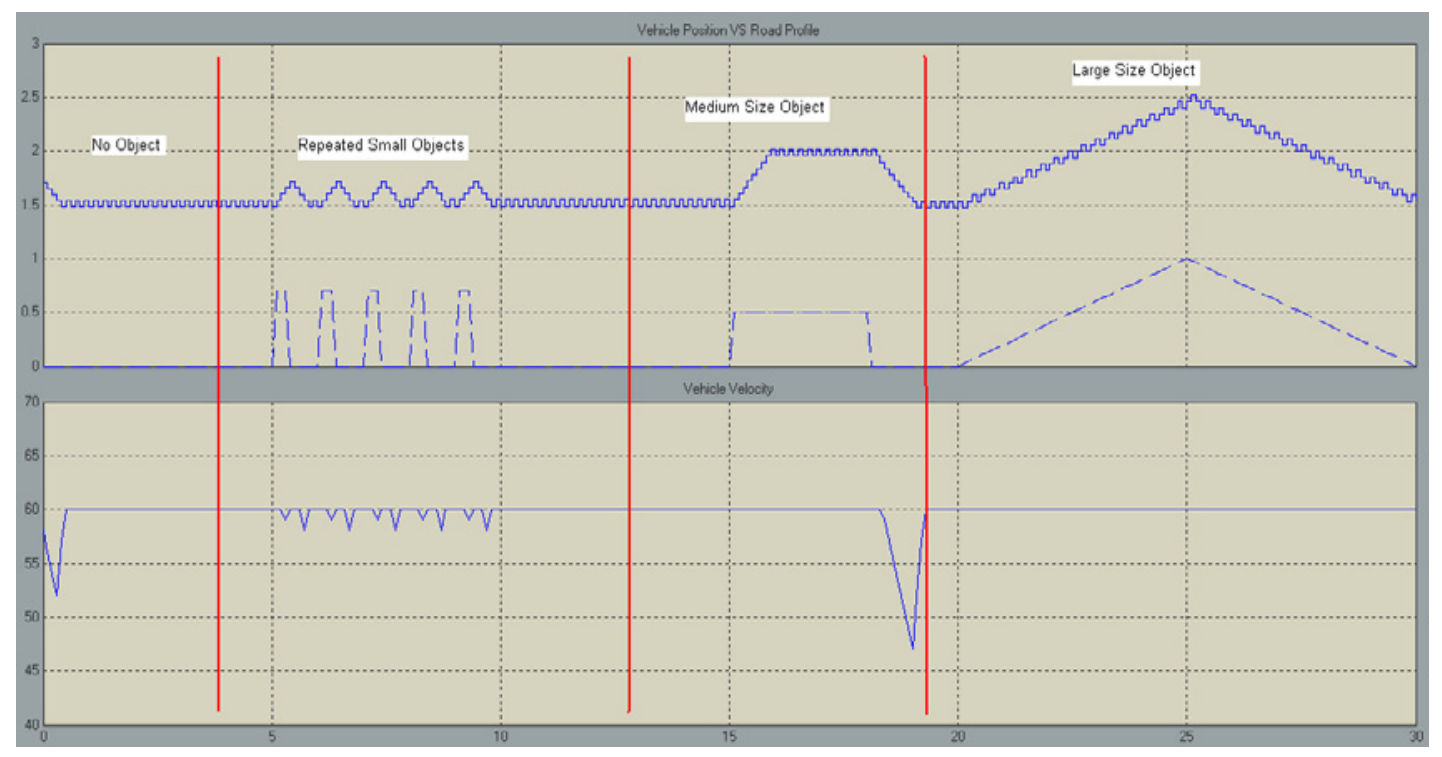

Figure 5. Simulation results for road with foreign object.

When the system is tested with the road profile with foreign object, the system give expected response to the foreign object. A closer look reveal that when there is a surge of big difference between the vehicle and the highway divider the system will reduce it speed due the constant action of steering system.

\section{Conclusion}

This paper has shown the possibilities of the AutoGuide system to be implemented in the real application. The system will enhance the highway road safety and is a step further in its goal of becoming an artificial driver. Yet the system is still a part of big ideas of realization of the actual system since there are still a lot of improvements that need to be done and the variable used by the current developed system is limited and simplified. The feasibilities of this system to be implemented can be shown by producing a prototype or similar miniature system for experimental value of actual data. This can further improve the level of understanding how this system functioning.

\section{References}

1. F. O. Jaksch, Int. J. of Veh. Des., 4 (1983)

2. E. Lissel, .P Andreas, R. Berghholz, R. Holze, VW AG Elec. Research, (1995)

3. M. A. Brackstone, M. McDonald, MIHT, MCIT, (1995)

4. T.A. Lasky, B. Ravani, AHMCT, UC Davis, (1994)

5 S. G. Kim, J. H. Kim, Int. J. of AT, 4, pg -173-180, (2003)

6. S. B. Modi, P. Chandak, V. S. Murty, E. L. Hall, Intelligent Robots and Computer Vision XX: Algorithm, Techniques, and Active Vision, (2001) 